\title{
Enhancing AFM process productivity through improved fixturing
}

\author{
R. S. Walia $\cdot$ H. S. Shan $\cdot$ P. Kumar
}

Received: 25 March 2008 / Accepted: 4 December 2008 / Published online: 4 February 2009

(C) The Author(s) 2009. This article is published with open access at Springerlink.com

\begin{abstract}
Abrasive flow machining (AFM) is a nonconventional finishing process that deburrs and polishes by forcing an abrasive laden media across the workpiece surface. The process embraces a wide range of applications from critical aerospace and medical components to highproduction volumes of parts. One serious limitation of this process is its low productivity in terms of rate of improvement in surface roughness. Limited efforts have hitherto been directed towards enhancing the productivity of this process with regard to better quality of workpiece surface. This paper discusses improved fixturing as a technique for productivity enhancement in terms of surface roughness $\left(R_{\mathrm{a}}\right)$. A rotating centrifugal-force-generating (CFG) rod is used inside the cylindrical workpiece which provides the centrifugal force to the abrasive particles normal to the axis of workpiece. The effect of the key parameters on the performance of process has been studied. The results shows that for a given improvement in $R_{\mathrm{a}}$ value, the processing time can be reduced by as much as $70-80 \%$. It is seen that the significant process parameters are revolutions per minute of $\mathrm{CFG}$ rod, extrusion pressure and abrasive mesh size.
\end{abstract}

Keywords AFM - CFAAFM - CFG · Rotational speeds . Surface roughness $\cdot$ ANOVA

R. S. Walia $\cdot$ H. S. Shan $\cdot$ P. Kumar $(\bowtie)$

Department of Mechanical and Industrial Engineering, Indian Institute of Technology,

Roorkee 247667, India

e-mail: kumarfme@iitr.ernet.in

R. S. Walia

e-mail: waliaravinder@yahoo.com

H. S. Shan

e-mail: shanhfme@iitr.ernet.in

\section{Introduction}

Modern metal working industry has several challenges such as to control costs, decrease lead time from design to production, improvement of product quality, and machining/finishing of difficult to machine materials. The most labour-intensive uncontrollable area in the manufacture of precision parts involves final machining (or finishing) operations. Finishing operations usually cost approximately $15 \%$ of the total machining cost in a production cycle $[1$, 2]. The marginal cost of surface finish increases sharply for a roughness value of $1 \mu \mathrm{m}$. Abrasive flow machining (AFM) is one of the processes capable of facing the aforementioned challenges. In this process, a specially formulated, pliable, plastic-like abrasive laden media is forced to flow over edges and surfaces to be deburred, polished or radiused. Machining action occurs whenever the abrasive laden media (elastic/viscoelastic polymer) pass through the restrictive passages. AFM uses two vertically opposed cylinders that extrude abrasive media back and forth through passages formed by the workpiece and tooling. Combination of these up and down strokes constitutes a process cycle. The process was developed earlier to find a more effective method of deburring hydraulic control blocks, which were initially being deburred by hand. The media contain a large number of random cutting edges with indefinite orientation and geometry for effective removal of material with chip sizes smaller than those obtained during machining using cutting tools with defined edges. Because of extremely thin chips produced in AFM, the process can produce better surface finish and closer tolerances, and it can generate more intricate surface features and machine harder and difficultto-machine materials [2]. The process has found many applications in the fields of aerospace, automotive, elec- 
tronic, die making, surgical implants, etc. and is being extensively used for removing recast layer from the surfaces generated by electro-discharge machining. AFM process can finish surfaces up to $0.05 \mu \mathrm{m}$, deburr holes as small as $0.2 \mathrm{~mm}$, radius edges from 0.025 to $1.5 \mathrm{~mm}$ and hole tolerance up to $\pm 5 \mu \mathrm{m}$ can be held [3]. It can offer as much as $90 \%$ time saving over hand finishing operations [4]. In the first few cycles, the major part of the total improvement in the surface finish occurs with a minimal dimensional change (usually 0.013 to $0.025 \mathrm{~mm}$ ). In order to cater to the requirement of high accuracy and high efficiency finishing of materials, AFM is gaining importance day by day. The AFM process has a limitation too with regard to achieving required surface finish. It is the time to achieve the required surface finish (cycle time). With the aim to overcome the difficulty of longer cycle time, the present paper reports the findings of a hybrid process which permits AFM to be carried out with additional centrifugal force applied onto the cutting media.

\section{Literature survey}

Extrusion pressure of media has been reported to significantly affect the work surface roughness but was found to fall just short of significance requirements with respect to material removal [4, 5]. Przyklenk [6] claimed its effect both on material removal and surface roughness as significant. At higher pressure, the improvement in material removal just tends to stabilise. The media flow rate has been reported to be a less influential parameter in respect to material removal [7]. The media viscosity and geometrical shape of the workpiece also affect the flow pattern. A number of studies $[6,8-12]$ have shown that abrasion is pronounced in some initial cycles, after which both material removal rate and improvement in surface roughness get stabilised. It has also been observed that the greater the reduction ratio, the more is the material removal from the workpiece for a specified number of cycles. There exists the possibility of using a large range of concentration of abrasive particles in carrier media (two to 12 times the
Fig. 1 Schematic illustration of the CFAAFM process setup: 1 Piston; 2 Abrasive laden media; 3 Sleeve; 4 Fixture; 5 Rotating CFG rod; 6 Workpiece; 7 Rotating attachment; 8 Bearing; 9 Idler gear; 10 Prime gear; 11 Sealing; 12 Media cylinder

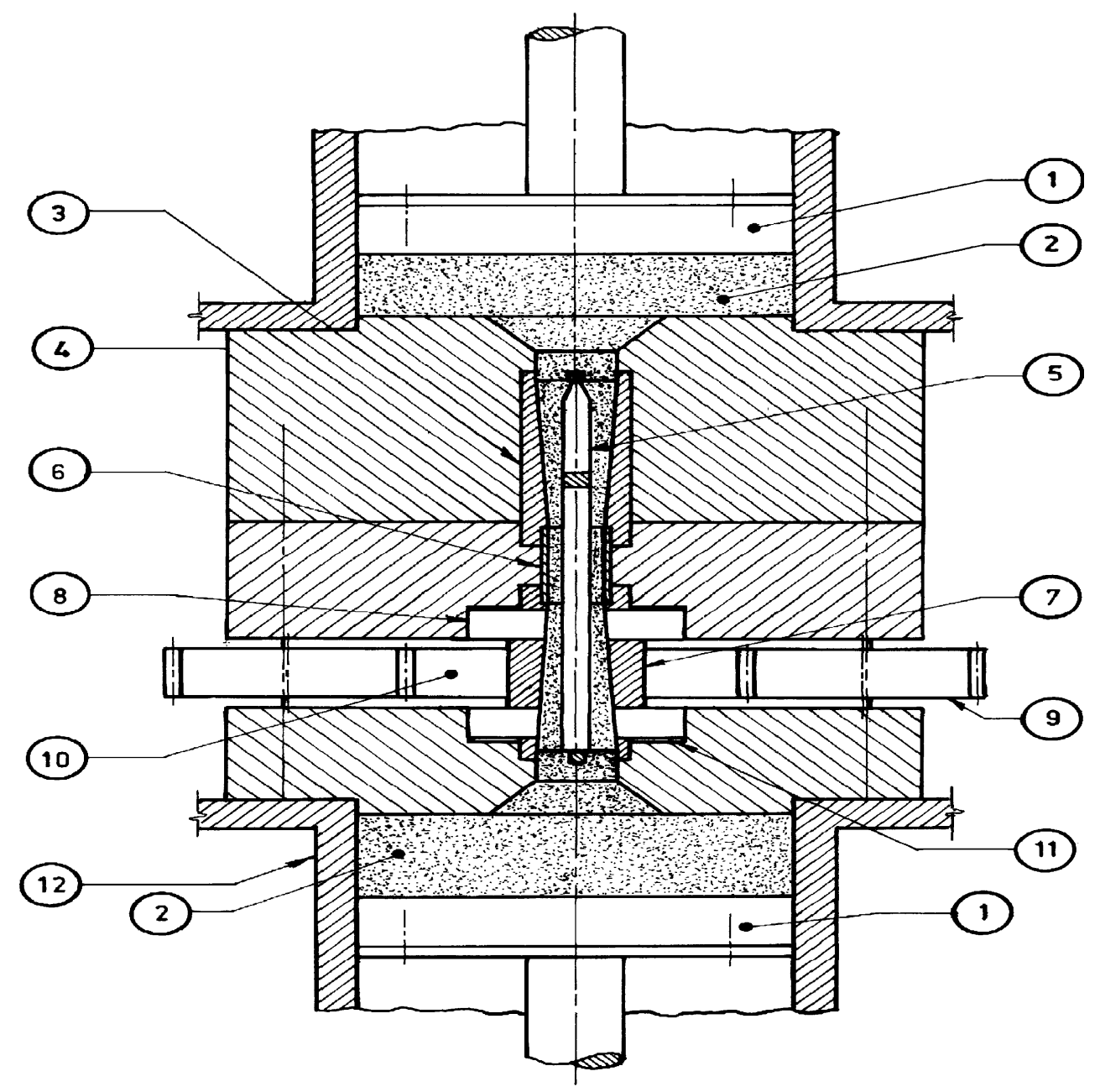


weight of carrier media) [15]. However, it has also been suggested that abrasive grain to base material ratio (by weight) should vary from $4: 1$ to $1: 4$, with $1: 1$ as the most appropriate ratio [13]. A rheological investigation [14] of polyborosiloxane media indicates that with increase in viscosity of media, pressure drop across the passage and processing time both increase, but the rate of temperature rise decreases.

The concept of hybrid machining processes (HMP) is currently gaining attention with the aim of achieving better performance of the modern machining processes. The underlying principle of hybrid machining processes is to club the advantages and avoid or reduce the adverse effects (if any) of the constituent processes [15]. An example of HMP is the ultrasonic flow polishing, which is the combination of AFM and ultrasonic machining [16]. Surface finish improvements of up to 10:1 have been recorded by the process. Another hybrid machining process is the orbital flow machining process, which utilises the principle of orbital grinding and AFM [17]. Still another example of HMP is the ultrasonic-assisted grinding for the special application of machining the ceramics [18]. The finishing of metals by magnetic abrasive machining (MAM) has been studied by many researchers [19-23]. The characteristic feature of MAM is that it employs very small machining pressure and is easily controllable with the help of input current to the electromagnet. Magnetically assisted abrasive flow machining, which is the combination of AFM and magnetic abrasive finishing (MAF), has been shown to give better results than obtained from individual AFM or MAF [24]. Literature survey indicates that though AFM has excellent characteristics as regards generation of super finish on inaccessible areas of workpiece surface, limited efforts have hitherto been directed towards improving the process efficiency of AFM. There appears to be a need for more research contribution to develop modification of this process which will give better quality surface economically. One such modification has been attempted in the present investigation. This involves increasing the pressure on the abrasive in the media whilst it contacts the surface of workpiece during processing. This has been achieved by providing a centrifugal-force-generating (CFG) rod with rotational mechanism in the AFM setup that helps the media to simultaneously rotate at a speed whilst being axially pushed. The rotation of the rod causes a centrifugal force to act on the media, which in turn increases media contact quality. Figure 1 shows the schematic of the setup designed and fabricated. The modified process has been termed as centrifugal-force-assisted abrasive flow machining (CFAAFM). This new process also helps to increase the reduction ratio for a given workpiece geometry compared to conventional AFM process.

\section{Centrifugal-force-assisted abrasive flow machining}

Figure 2 shows the modified fixture employed for the CFAAFM process. The fixture is attached to the indigenously made AFM setup in Machine Tool Laboratory at Indian Institute of Technology, Roorkee. It holds the workpiece which is placed in between the media cylinders to create an artificial dead zone and increase the pressure required for extruding the media. The fixture is made in three parts and the workpiece and rotating attachment are placed in between the three parts. The media containing the abrasive particles flows from one cylinder to the other cylinder through the central hole in the workpiece. The workpiece is surrounded by an attachment specially designed to give necessary rotary motion to CFG rod. Whilst the media flow through the workpiece cavity, the rotation of the CFG rod causes a centrifugal force to act on the abrasive particles so as to throw them on the internal surface (normal to the axis) of the workpiece. In CFAAFM, the media are thus subjected to the extrusion pressure as well as to the additional centrifugal pressure. Media used for the present investigation consist of a silicon-based polymer, hydrocarbon gel and abrasive particles. The polymer was prepared by a special technique for which a patent was granted. The gel was prepared by reacting aluminium stearate with hydrocarbon oil. The gel was then mixed into the polymer in a suitable proportion by vigorous kneading. The mixture of the polymer and gel was used as a carrier compound in the media. In the present work, the abrasive particles called Brown Super Emery (trade name) were used. It is one of the natural abrasives, which is the impure form of corundum.

\subsection{Experimentation}

In the preliminary set of experiments, brass as workpiece material was used. The test workpieces $\varphi 10 \times 16$-mm-long cylindrical parts with central hole bored to $8.00 \pm 0.05-\mathrm{mm}$

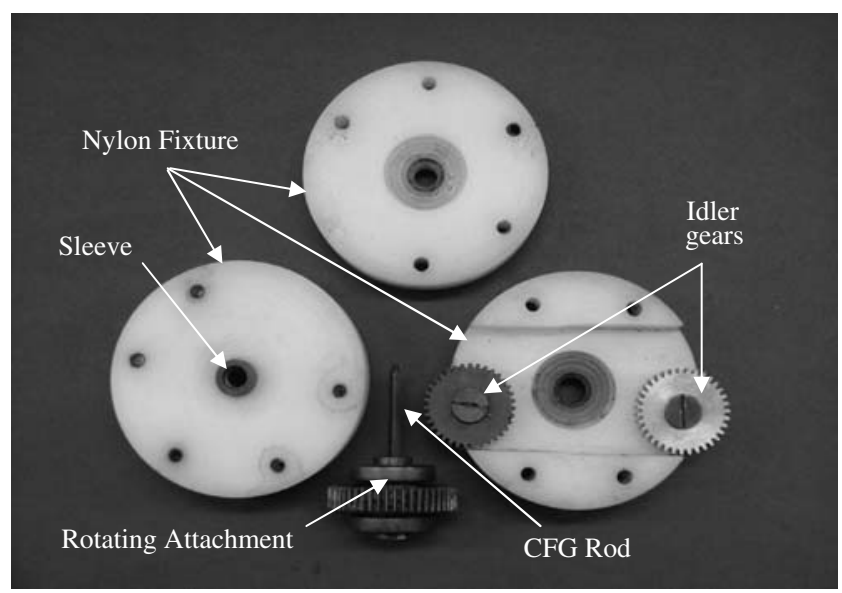

Fig. 2 Details of CFAAFM fixture and rotating attachment 
diameter as shown in Figs. 2 and 3 were prepared. The internal cylindrical surface of the workpieces was finishedmachined by CFAAFM at different rotational speeds of CFG rod. Each workpiece was machined for a predetermined number of cycles. The workpiece was taken out from the setup and cleaned with acetone before any measurement was taken. Only those specimens whose initial surface roughness of the hole was in a quite narrow range were selected for the experiments so as to avoid any extrageneous effect on the response parameters. Before and after processing a test specimen, the surface roughness $\left(R_{\mathrm{a}}\right)$ was measured at 25 random locations all over the hole surface in the axial direction on the internal cylindrical surface using a Mahr perthometer. The average of $R_{\mathrm{a}}$ values was calculated and the percentage improvement in roughness $\Delta R_{\mathrm{a}}$ was estimated as:

$\Delta R_{\mathrm{a}}=\frac{\text { (initial roughness }- \text { roughness after machining }) \times 100}{\text { initial roughness }}$.

In the present study, $\mathrm{L}_{9}$ Taguchi orthogonal array is used [26]. The surface roughness data were analysed to determine the effect of the various design parameters. The experimental results are then transformed into signal-to-noise $(\mathrm{S} / \mathrm{N})$ ratio. Taguchi recommends the use of $\mathrm{S} / \mathrm{N}$ ratio to measure the quality characteristics deviating from the desired values. Usually, there are three categories of quality characteristic in the analysis of the $\mathrm{S} / \mathrm{N}$ ratio, viz. the lower-the-better, the higher-the-better and the nominal-the-better. The $\mathrm{S} / \mathrm{N}$ ratio for each set of experimentation is computed. Regardless of the category of the quality characteristic, a greater $\mathrm{S} / \mathrm{N}$ ratio corresponds to better quality characteristics. Therefore, the optimal level of the process parameters is the level with the greatest $\mathrm{S} / \mathrm{N}$ ratio. The quality characteristic for percentage improvement in surface roughness is taken as of higher-thebetter type. The $\mathrm{S} / \mathrm{N}$ ratio for the higher-the-better type of response can be computed $[25,27]$ as:

$(\mathrm{S} / \mathrm{N})_{\mathrm{HB}}=-10 \log \left[\frac{1}{R} \sum_{j=1}^{R}\left(1 / Y_{j}^{2}\right)\right]$

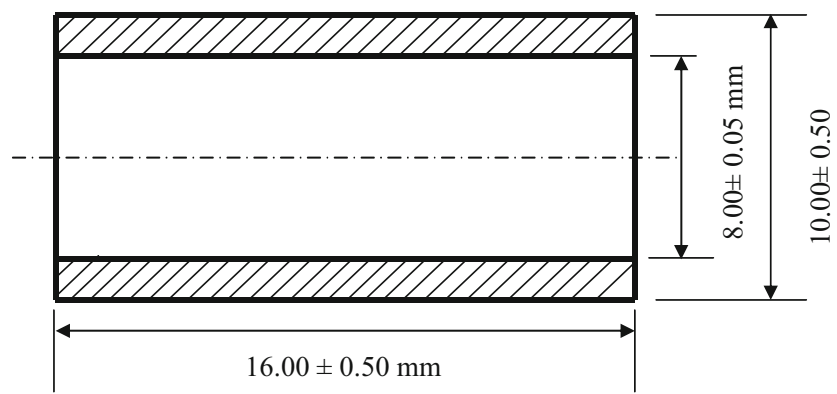

Fig. 3 Test piece

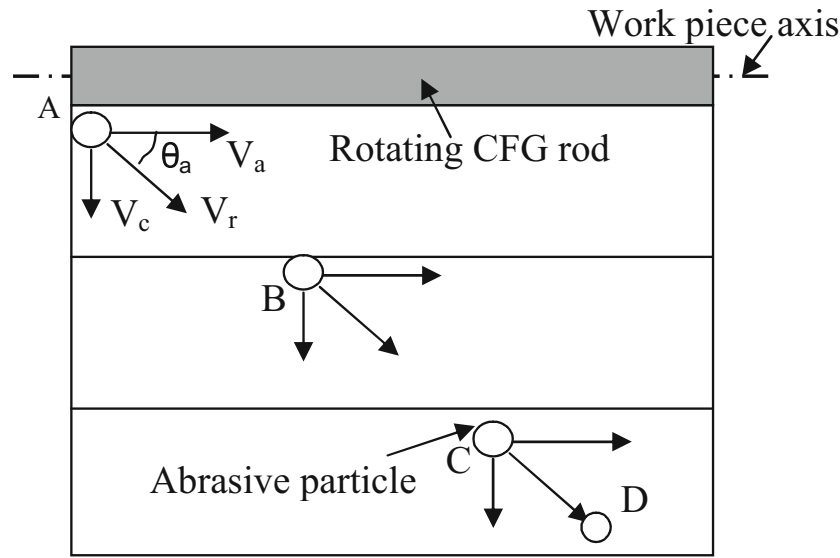

Work piece inner surface

Fig. 4 Deflection of abrasive particle in CFAAFM process

where $Y_{j}, j=1,2, \ldots n$ are the response values under the trial conditions repeated $R$ times.

Analysis of variance (ANOVA) has been performed to identify the process parameters that are statistically significant. With the S/N and ANOVA analyses, the optimal combination of the process parameters has been predicted. The following parameters were selected to investigate their effect on percentage improvement in surface roughness in CFAAFM process: rotational speeds of CFG rod, extrusion pressure and abrasive grits size. The following parameters were selected constant during the entire experimentation: polymer to gel ratio, 4:3; abrasive concentration, 1:3; workpiece material, brass; number of cycle, 3; abrasive type, $\mathrm{Al}_{2} \mathrm{O}_{3}$ and temperature, $32 \pm 2^{\circ} \mathrm{C}$. The design parameters as well as their chosen levels considered for the Taguchi experiment are listed in Table 1. The experiments were conducted at each trial conditions as given in Table 2. For each trial, experiments were replicated (three times). The estimated percentage improvement in surface roughness and signal-to-noise ratio are given in Table 2.

Table 1 Factors and their levels

\begin{tabular}{llccc}
\hline $\begin{array}{l}\text { Process parameter } \\
\text { designation }\end{array}$ & Process parameters & $\begin{array}{l}\text { Level } \\
1\end{array}$ & $\begin{array}{l}\text { Level } \\
2\end{array}$ & \multicolumn{2}{l}{$\begin{array}{l}\text { Level } \\
3\end{array}$} \\
\hline$A$ & $\begin{array}{l}\text { Rotational speed of } \\
\text { CFG rod (rpm) }\end{array}$ & 0 & 35 & 70 \\
$B$ & $\begin{array}{c}\text { Extrusion pressure } \\
\text { (bar) }\end{array}$ & 20 & 27 & 34 \\
$C$ & Grit size $(\mu \mathrm{m})$ & 250 & 150 & 100 \\
\hline
\end{tabular}

Polymer to gel ratio, 4:3; abrasive concentration, 1:3; workpiece material, brass; number of cycle, 3 ; abrasive type, $\mathrm{Al}_{2} \mathrm{O}_{3}$; temperature, $32 \pm 2^{\circ} \mathrm{C}$ 
Table 2 Orthogonal array for L9 with responses (raw data and S/N ratios)

\begin{tabular}{|c|c|c|c|c|c|c|c|}
\hline \multirow[t]{2}{*}{ Trial no. } & \multicolumn{3}{|l|}{ Input parameters } & \multicolumn{3}{|c|}{ Responses (raw data) $\Delta R_{\mathrm{a}}$} & \multirow[t]{2}{*}{$\mathrm{S} / \mathrm{N}$ ratio $(\mathrm{dB})$} \\
\hline & $\begin{array}{l}\text { Rotational speed of CFG rod (rpm) } \\
A \\
\text { Trial conditions }\end{array}$ & $\begin{array}{l}\text { Extrusion pressure (bar) } \\
B\end{array}$ & $\begin{array}{l}\text { Grit size }(\mu \mathrm{m}) \\
C\end{array}$ & $\mathrm{R} 1$ & $\mathrm{R} 2$ & R3 & \\
\hline 1 & 0 & 20 & 250 & 1.38 & 1.19 & 1.58 & 0.88 \\
\hline 2 & 0 & 27 & 150 & 1.89 & 1.97 & 1.73 & 3.61 \\
\hline 3 & 0 & 34 & 100 & 2.46 & 2.41 & 2.12 & 5.53 \\
\hline 4 & 35 & 20 & 150 & 4.35 & 4.04 & 4.83 & 11.05 \\
\hline 5 & 35 & 27 & 100 & 5.96 & 5.99 & 5.57 & 13.55 \\
\hline 6 & 35 & 34 & 250 & 5.09 & 5.46 & 4.59 & 12.23 \\
\hline 7 & 70 & 20 & 100 & 8.59 & 8.76 & 8.86 & 17.06 \\
\hline 8 & 70 & 27 & 250 & 8.29 & 8.12 & 8.31 & 16.56 \\
\hline 9 & 70 & 34 & 150 & 9.39 & 9.22 & 9.45 & 17.66 \\
\hline Total & & & & 47.4 & 47.16 & 47.04 & 98.13 \\
\hline
\end{tabular}

$\mathrm{R} 1, \mathrm{R} 2$ and R3 represent percentage improvement in surface roughness value for three repetitions of each trial. $\bar{T}$ is the grant average of percentage improvement in surface roughness $=(47.4+47.16+47.04) / 27=5.24$. Polymer to gel ratio, $4: 3$; abrasive concentration, $1: 3$; workpiece material, brass; number of cycle, 3 ; abrasive type, $\mathrm{Al}_{2} \mathrm{O}_{3}$

\section{Abrasive particle attack in CFAAFM process}

In AFM, the abrasive particles are flowing along with the polymer-based media in the axial direction through the workpiece passage. When $\mathrm{CFG}$ rod rotates inside the hollow cylindrical workpiece, it throws the abrasive laden media coming in its contact to move in a circular path. As a result, centrifugal force acts on the abrasive particles. This force forces the abrasive particle to move towards direction normal to the axis of workpiece, as shown in Figs. 4.

Consider an abrasive particle of mass " $m$ " enters the workpiece passage. During its flow through the workpiece passage, it is acted upon by the following two forces:

1. $F_{\mathrm{a}}$ : axial thrust produced by movement of piston in the main cylinder;

2. $\quad F_{\mathrm{c}}$ : centrifugal force due to circular motion caused by rotation of CFG rod. This acts in direction normal to the flow of media through the workpiece passage.

$F_{\text {a }}$ causes the abrasive particle to move in axial direction with velocity $V_{\mathrm{a}}$, whilst $F_{\mathrm{c}}$ tends to push it normal to the workpiece axis (towards the inner surface of workpiece) with velocity $V_{\mathrm{c}}$.

$F_{\mathrm{c}}$ acting on an abrasive particle is given as:

$F_{\mathrm{c}}=m \omega^{2} r$

where $\omega$ is angular velocity of the abrasive particle imparted to it due to CFG rod rotation, and $r$ is the radius of path in which the abrasive particle is rotating.

Under the influence of $F_{\mathrm{c}}$, the axially flowing abrasive particles in the media start moving towards the inner surface of workpiece. In this condition, the viscous force will be developed which is more predominant than the inertia force acting on the abrasive particles. Thus, an opposite frictional drag force $\left(F_{\mathrm{drag}}\right)$ will act on the particles as given by Stokes law [13]:

$F_{\text {drag }}=3 \pi \mu d_{\mathrm{p}} V_{\mathrm{c}}$

where $\mu$ is the viscosity of media and $d_{\mathrm{p}}$ is the diameter of abrasive particle.

Assume $F_{\mathrm{c}}$ to be constant over the small time step (dt), where $t$ denotes the elapsed time for abrasive particle flow in the abrasive laden media. $V_{\mathrm{c}}$ can be found from the following equation:

$F_{\mathrm{c}}-F_{\mathrm{drag}}=m \frac{\mathrm{d} V_{\mathrm{c}}}{\mathrm{d} t}$

$F_{\mathrm{c}}-3 \pi \mu d_{\mathrm{p}} V_{\mathrm{c}}=m \frac{\mathrm{d} V_{\mathrm{c}}}{d \mathrm{t}}$.

Integrating and rearranging,

$\int \frac{\mathrm{d} V_{\mathrm{c}}}{F_{\mathrm{c}}-3 \pi \mu d_{\mathrm{p}} V_{\mathrm{c}}}=\frac{1}{m} \int \mathrm{d} t$

$\frac{\operatorname{In}\left(F_{\mathrm{c}}-3 \pi \mu d_{\mathrm{p}} V_{\mathrm{c}}\right)}{-3 \pi \mu d_{\mathrm{p}}}=\frac{t}{m}+k$

where $k$ is constant of integration. Applying boundary condition at $t=0, V_{\mathrm{c}}=0$

$\Rightarrow \quad k=\frac{\operatorname{In} F_{\mathrm{c}}}{-3 \pi \mu d_{\mathrm{p}}}$.

Hence,

$\frac{\operatorname{In}\left(F_{\mathrm{c}}-3 \pi \mu d_{\mathrm{p}} V_{\mathrm{c}}\right)}{-3 \pi \mu d_{\mathrm{p}}}=\frac{t}{m}+\frac{\operatorname{In} F_{\mathrm{c}}}{-3 \pi \mu d_{\mathrm{p}}}$

$F_{\mathrm{c}}-3 \pi \mu d_{\mathrm{p}} V_{\mathrm{c}}=e^{\Delta C}$

where $\Delta C=-3 \pi \mu d_{\mathrm{p}}\left(\frac{t}{m}+\frac{\operatorname{In} F_{\mathrm{c}}}{-3 \pi \mu d_{\mathrm{p}}}\right)$.

It is to be noted that the values of the mass of abrasive particle $\left(m=2.6 \times 10^{-7} \mathrm{~kg}\right)$ and media viscosity $(\mu=$ 


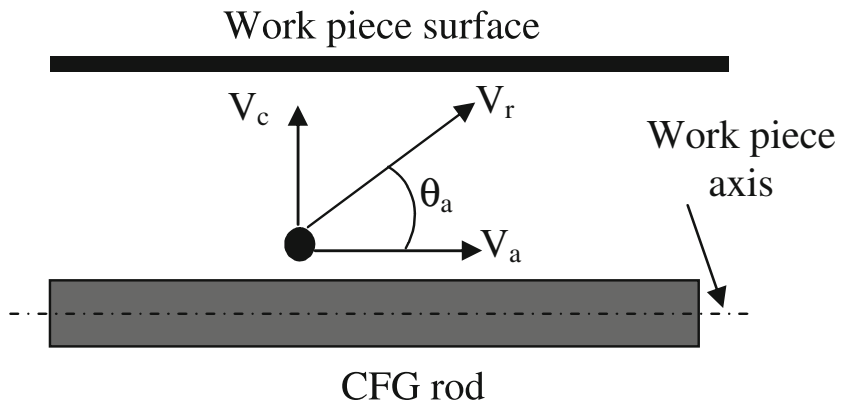

Fig. 5 Simulating the path of abrasive particle in CFAAFM process. a Effect of rotational speeds on raw data. b Effect of extrusion pressure on raw data. c Effect of abrasive grits size on raw data. Figure 4 Response curves: polymer to gel ratio, 4:3; number of cycles, 3; abrasive concentration, 1:3; workpiece material, brass; abrasive, $\mathrm{Al}_{2} \mathrm{O}_{3}$

$414 \mathrm{~Pa} \mathrm{~s}$ ) make the term $\Delta C$ considerably high. Therefore, Eq. 5 can be written as:

$F_{\mathrm{c}}-3 \pi \mu d_{\mathrm{p}} V_{\mathrm{c}} \cong 0$.

Hence

$V_{\mathrm{c}}=\frac{F_{\mathrm{c}}}{3 \pi \mu d_{\mathrm{p}}}$.

The value of $V_{\mathrm{a}}$ can be estimated from extrusion pressure. The resultant velocity, $V_{\mathrm{r}}$, of abrasive particle due to $V_{\mathrm{a}}$ and $V_{\mathrm{c}}$ is given by:

$V_{\mathrm{r}}=\sqrt{\left(V_{\mathrm{a}}^{2}+V_{\mathrm{c}}^{2}\right)}$

and

$\theta_{\mathrm{a}}=\tan ^{-1}\left(\frac{V_{\mathrm{c}}}{V_{\mathrm{a}}}\right)$

where $\theta_{\mathrm{a}}$ defines the direction of movement of the abrasive particle with which it strikes the cavity surface (called angle of attack). Figure 5 illustrates the model proposed for depicting the path of an abrasive particle in the workpiece passage under the influence of thrust and centrifugal force. The mark ' $\mathrm{A}$ ' represents the entry point of the abrasive particle in the workpiece cavity. As the abrasive particle travels through the workpiece passage, it is acted upon by thrust and centrifugal forces, causing it to travel with velocity $V_{\mathrm{r}}$. The direction and magnitude of $V_{\mathrm{r}}$ change continuously as the particle moves towards the cavity surface, to the position ' $\mathrm{B}$ ' and then to ' $\mathrm{C}$ '. At ' $\mathrm{B}$ ', $V_{\mathrm{a}}$ is smaller than what it was at ' $A$ ' due to the presence of boundary layer phenomenon, but $V_{\mathrm{c}}$ will depend upon the angular rotational speed and the radius of path of its rotation. The resultant velocity acting on the particle at position ' $\mathrm{B}$ ' causes it to move towards the position ' $C$ '. This movement of the abrasive particle due to varying resultant velocity ultimately makes it to strike at the cavity surface. The angle of strike of the abrasive particle can have important influence on the MR and $\Delta R_{\mathrm{a}}$, as it affects the mechanism of material removal from the surface of cavity.

\section{Results and discussions}

The independent process parameters selected for this study were rotational speed of CFG rod, extrusion pressure and abrasive grit size used in the media, whilst other parameters remained constant. The average value of $\mathrm{S} / \mathrm{N}$ ratio $(\mathrm{dB})$ of percentage improvement in surface roughness and that of raw data at levels L1, L2 and L3 for each parameter are shown in Tables 3 and 4, respectively. The effect of the individual parameters on average value of $\Delta R_{\mathrm{a}}$ as well as on $\mathrm{S} / \mathrm{N}$ ratio $(\mathrm{dB})$ is shown in Fig. 5. From the trend of response curves for $\Delta R_{\mathrm{a}}$ at different levels of the process parameters, it can be observed that when rotational speed of the CFG rod increases, $\Delta R_{\mathrm{a}}$ improves. The possible reason for this result is that those abrasive particles flowing with the media which would otherwise have gone without touching the workpiece surface (case of AFM) change their path of flow and strike the inner surface of workpiece at an inclination when acted upon by a centrifugal force (due to rotation of CFG rod in the CFAAFM). The greater the rotational speed of rod, the higher is the pressure and the greater is the $\Delta R_{\mathrm{a}}$. The centrifugal force also enhances the number

Table 3 Main effects ( $\mathrm{S} / \mathrm{N}$ data)

\begin{tabular}{|c|c|c|c|c|}
\hline Process parameter & Level & Rotational speed of CFG rod (rpm) $(A)$ & Extrusion pressure $(B)$ & Grit size $(C)$ \\
\hline \multirow{3}{*}{ Average values $\left(\% R_{\mathrm{a}}\right)$} & L1 & 3.34 & 9.67 & 9.89 \\
\hline & $\mathrm{L} 2$ & 12.28 & 11.24 & 10.77 \\
\hline & L3 & 17.09 & 11.81 & 12.05 \\
\hline \multirow[t]{3}{*}{ Main effects $\left(\% R_{\mathrm{a}}\right)$} & $\mathrm{L} 2-\mathrm{L} 1$ & 8.94 & 1.57 & 0.88 \\
\hline & $\mathrm{L} 3-\mathrm{L} 2$ & 4.81 & 0.57 & 1.28 \\
\hline & Difference $\{(\mathrm{L} 3-\mathrm{L} 2)-(\mathrm{L} 2-\mathrm{L} 1)\}$ & -4.13 & -1.00 & 0.4 \\
\hline
\end{tabular}

$\mathrm{L} 1, \mathrm{~L} 2$ and L3 represent average values of $\mathrm{S} / \mathrm{N}$ ratio at levels 1, 2 and 3, respectively, of parameters. L2-L1 is the average main effect when the corresponding parameter changes from level 1 to level 2. L3-L2 is the average main effect when the corresponding parameter changes from level 2 to level 3 
Table 4 Main effects (raw data)

\begin{tabular}{|c|c|c|c|c|}
\hline Process parameter & Level & Rotational speed of CFG rod (rpm) $(A)$ & Extrusion pressure $(B)$ & Grit size $(C)$ \\
\hline \multirow[t]{3}{*}{ Average values $\left(\% R_{\mathrm{a}}\right)$} & L1 & 1.86 & 4.84 & 4.89 \\
\hline & $\mathrm{L} 2$ & 5.10 & 5.31 & 5.21 \\
\hline & L3 & 8.78 & 5.58 & 5.64 \\
\hline \multirow[t]{3}{*}{ Main effects $\left(\% R_{\mathrm{a}}\right)$} & $\mathrm{L} 2-\mathrm{L} 1$ & 3.24 & 0.47 & 0.32 \\
\hline & $\mathrm{L} 3-\mathrm{L} 2$ & 3.68 & 0.26 & 0.43 \\
\hline & Difference $\{(\mathrm{L} 3-\mathrm{L} 2)-(\mathrm{L} 2-\mathrm{L} 1)\}$ & 0.44 & -0.21 & 0.11 \\
\hline
\end{tabular}

L1, L2 and L3 represent average values raw data at levels 1, 2 and 3, respectively, of parameters. L2-L1 is the average main effect when the corresponding parameter changes from level 1 to level 2. L3-L2 is the average main effect when the corresponding parameter changes from level 2 to level 3

Fig. 6 Axisymmetric view of rolling and ploughing action of abrasive grain

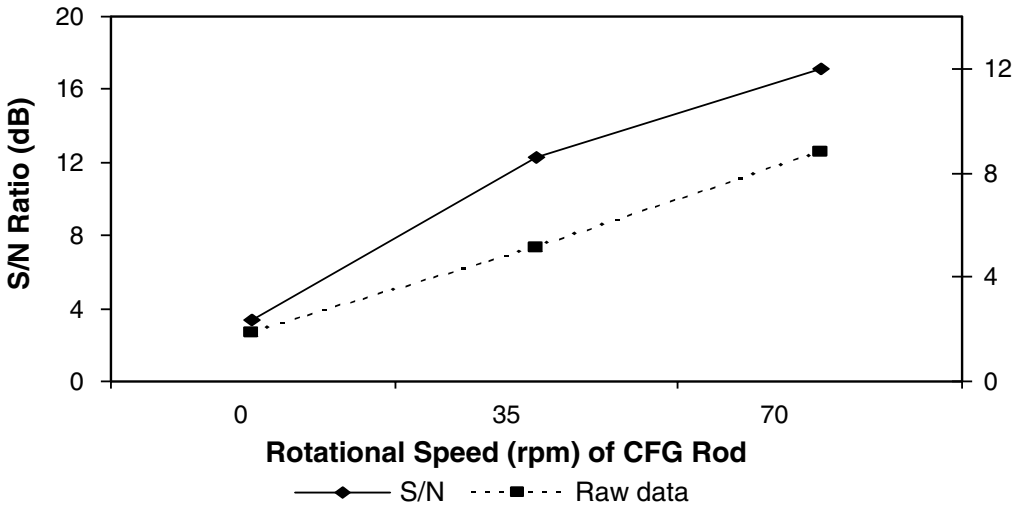

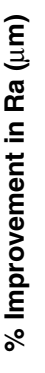

(a) Effect of Rotational Speeds on Raw Data

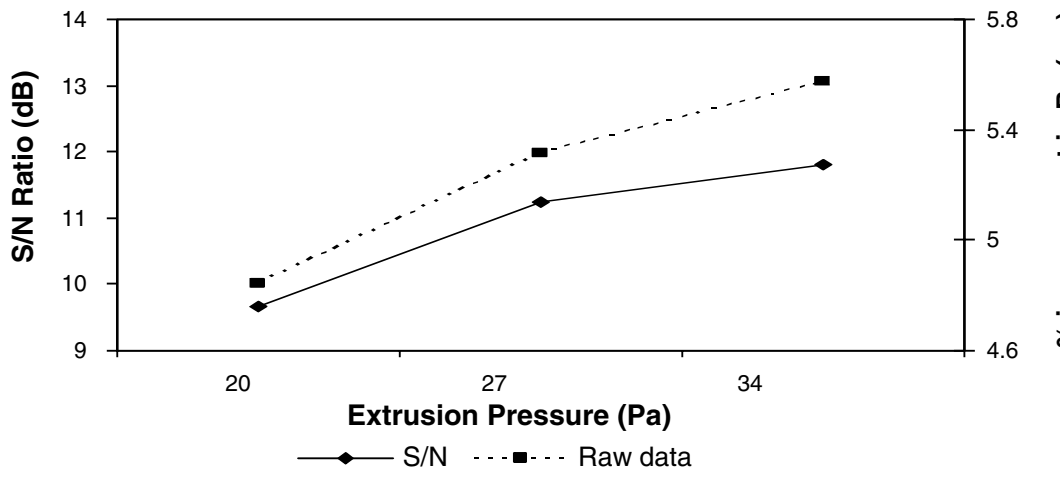

(b) Effect of Extrusion Pressure on Raw Data

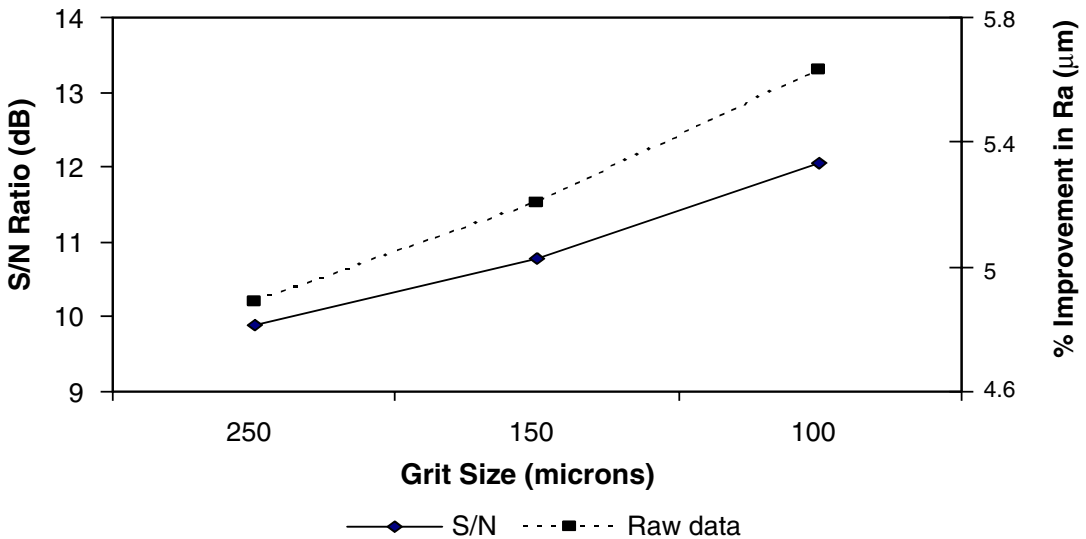

(c) Effect of Abrasive Grits Size on Raw Data 
of active abrasive grits taking parts in abrasion with the result that more abrasive particles progressively abrade all the high metal peaks on the work surface, producing a fine finish in fewer cycles.

Figure 5a depicts the variation of $\Delta R_{\mathrm{a}}$ with respect to the rotational speed of CFG rod for a given numbers of cycles. These results imply that fewer cycles are required to reach the given value of surface roughness in CFAAFM process as compared to the conventional AFM process. It can be observed from Fig. $5 \mathrm{~b}$ that the increase in extrusion pressure improves the work surface finish. Within the range of extrusion or axial pressure applied, the higher the pressure, the greater is the improvement in surface finish, which tends to stabilise beyond a certain level. Higher axial pressure enables the abrasive particles to roll on the surface with more force, resulting in faster removal of metal peaks on the work surface and hence rapid achievement of the required surface finish. The centrifugal force acts on the abrasive particles in normal direction, whilst the extrusion pressure provides the axial pressure on the abrasive particles. As such, rolling of the abrasive grains is primarily due to the extrusion pressure, whilst the indenting action is primarily due to centrifugal force. However, an abrasive grain embedded in the workpiece due to higher resultant pressure (due to combined action of axial and centrifugal force) may be 'thrown' out by the subsequent abrasive grains. As the embedded abrasive grain is dislodged, it chips away a portion of the workpiece, leading to ploughing action (Fig. 6). The total number of active grains shall increase many folds as the abrasive particles are thrown from the CFG rod to the inner surface of the workpiece. This leads to replenishment of abrasive grains at the workpiece-abrasive media interface. The number of abrasive grains taking part in machining can be calculated by the procedure given in [28]. A major disadvantage of increasing the extrusion pressure as compared to the centrifugal force is that in this, only very few abrasive particles (which are near to work surface) participate in abrading whilst the remaining particles pass through the workpiece without performing any cutting action. Further,

Table 5 Pooled ANOVA (raw data)

\begin{tabular}{lrllllr}
\hline Source & SS & $d f$ & $V$ & $F$ ratio & \multicolumn{1}{l}{ SS } & \multicolumn{1}{c}{$P \%$} \\
\hline Rotation speed (rpm) & 215.64 & 2 & 107.82 & $913.71^{\mathrm{a}}$ & 215.40 & 96.59 \\
Extrusion pressure & 2.49 & 2 & 1.25 & $10.57^{\mathrm{a}}$ & 2.26 & 1.01 \\
Grit size & 2.52 & 2 & 1.26 & $10.68^{\mathrm{a}}$ & 2.28 & 1.02 \\
$E$ (pooled error) & 2.36 & 20 & 0.12 & & 3.07 & 1.38 \\
Total (T) & 223.01 & 26 & - & - & 223.01 & 100 \\
\hline
\end{tabular}

$S S$ sum of squares, $d f$ degree of freedom, $V$ variance, $S S$ ' pure sum of squares

${ }^{a}$ Significant at $95 \%$ confidence level
Table 6 Pooled ANOVA (S/N data)

\begin{tabular}{lrlrrrr}
\hline Source & \multicolumn{1}{l}{ SS } & $d f$ & & $F$ ratio & \multicolumn{1}{l}{ SS } & \multicolumn{1}{c}{$P \%$} \\
\hline Rotation speed (rpm) & 292.22 & 2 & 146.11 & $1461.52^{\mathrm{a}}$ & 292.02 & 95.17 \\
Extrusion pressure & 7.37 & 2 & 3.69 & $36.88^{\mathrm{a}}$ & 7.17 & 2.34 \\
Grit size & 7.06 & 2 & 3.53 & $35.32^{\mathrm{a}}$ & 6.86 & 2.24 \\
E (pooled error) & 0.20 & 2 & 0.10 & & 0.80 & 0.26 \\
Total $(T)$ & 306.85 & 8 & & & 306.85 & 100 \\
\hline
\end{tabular}

$S S$ sum of squares, $d f$ degree of freedom, $V$ variance, $S S$ ' pure sum of squares

${ }^{\text {a }}$ Significant at $95 \%$ confidence level

the same abrasive particles would abrade the whole length of workpiece, with the possible result that cutting edges of those abrasive particles get worn out which further reduce the cutting action [29-31]. From Fig. 5c, it can be noted that the use of finer grain size of the abrasive particles results in greater improvement of surface roughness. The reason for this seems obvious as with the constant mass ratio of abrasive-to-media used; the more number of finer grains would make finer but more number of cuts on the high spots of the work surface, consequently generating a smoother surface.

Figure $5 \mathrm{a}-\mathrm{c}$ indicates that all the parameters, rotational speed of CFG rod $(A)$, extrusion pressure $(B)$, and grit size (C) affect the $\mathrm{S} / \mathrm{N}$ ratio of both the mean and variation in the $\Delta R_{\mathrm{a}}$. It can be seen that the third level of each of the parameters, i.e. $\left(A_{3}\right),\left(B_{3}\right)$ and $\left(C_{3}\right)$, may provide maximum value of the percentage improvement in surface roughness. As in the case of all the parameters $A, B$ and $C$, the highest values of mean response correspond to the highest values of $\mathrm{S} / \mathrm{N}$ ratio.

In order to study the significance of the parameters, ANOVA was performed. The pooled ANOVA for raw data,

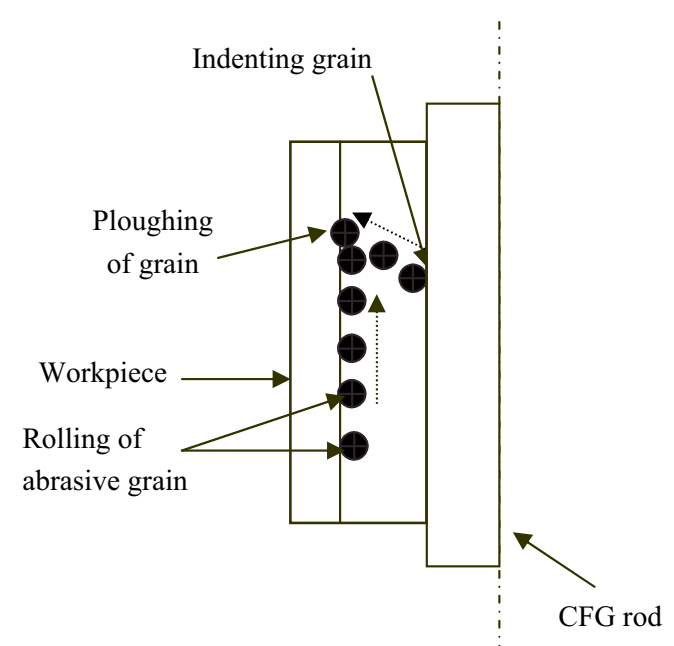

Fig. 7 a Two-dimensional contour plot. b Three-dimensional contour plot: (i) Before machining, (ii) At $0 \mathrm{rpm}$ (iii) At $35 \mathrm{rpm}$ and (iv) At $70 \mathrm{rpm}$ of $\mathrm{CFG}$ rod. Polymer to gel ratio, 4:3; abrasive concentration, 1:3; abrasive, $\mathrm{Al}_{2} \mathrm{O}_{3}$; extrusion pressure, 27 bar; number of cycles, 3 
i.e. percentage improvement in surface roughness values $\left(\Delta R_{\mathrm{a}}\right)$, is given in Table 5. The ANOVA was also performed for $\mathrm{S} / \mathrm{N}$ data and the pooled version is given in Table 6. From these tables, it can be observed that the parameters $A, B$ and $C$ significantly affect the percentage improvement in the surface roughness as well as the $\mathrm{S} / \mathrm{N}$ ratio. From these tables, it can also be noted that the percentage contribution is highest for $A, 96.59 \%$ for raw data and $95.17 \%$ for $\mathrm{S} / \mathrm{N}$ ratio.

The contour plots of surface roughness using optical surface profilers of workpiece processed at different rotational speed of CFG rod are presented as i-iv of Fig. 7. A visual inspection shows that there is not much difference between the surface roughness shown by twodimensional and three-dimensional contour plots. Thus, it can be inferred from these plots that the effect of process parameters on surface roughness is the same in both twoand three-dimension directions.

\section{Conclusions}

A centrifugal force has been applied with the help of rotation given to a CFG rod inside the hollow cylindrical workpiece being processed by CFAAFM, and increase in percentage improvement in surface roughness has been achieved. The following conclusions can be drawn from the study:

- It is possible to enhance the productivity of AFM by improved fixturing, i.e. with the introduction of $\mathrm{CFG}$ rod and providing rotation to it.

- Further enhancement in surface roughness can be expected with higher resultant pressure. However, the magnitudes of axial force and centrifugal force need to be optimised.

- Experiments using orthogonal array technique on brass test pieces conform that after a specific number of cycles, better surface finish is produced in CFAAFM as compared to conventional AFM.

- Analysis of results conform that the order of significant factors in decreasing trend are rotational speed of $\mathrm{CFG}$ rod, extrusion pressure and grit size.

- The results of analysis of SEM micrographs are in line with the experimental findings.

Open Access This article is distributed under the terms of the Creative Commons Attribution Noncommercial License which permits any noncommercial use, distribution, and reproduction in any medium, provided the original author(s) and source are credited.

\section{References}

1. Rhoades LJ (1988) Abrasive flow machining. Manufacturing Engineering, pp 75-78 (Nov)
2. Jain VK (2002) Advanced machining processes. Allied Publishers, New Delhi

3. Jain RK, Jain VK (1999) Abrasive fine finishing processes-a review. Int J Manuf Sci Prod 2(1):61-69

4. Sehijpal Singh (2002) Studies in metal finishing with magnetically assisted abrasive flow machining. PhD thesis, IIT, Roorkee

5. Williams RE, Rajurkar KP, Rhoades LJ (1989) Performance characteristics of abrasive flow machining. SME technical paper no. FC 89-806

6. Przyklenk (1986) Abrasive flow machining: a process for surface finishing and deburring of workpiece with a complicated shape by means of an abrasive laden medium. Advances in non-traditional machining, PED vol. 22 ASME, pp 101-110

7. Rhoades LJ (1985) Abrasive flow machining and its use. Proceedings of Non-Traditional Machining Conference, Cincinnati, $\mathrm{OH}, \mathrm{pp} 111-120$

8. Shan HS, Dubey AK (1997) Micro machining by flow of abrasives. Proceedings 17th AIMTDR Conference, Warangal, India, pp 269-275

9. Williams RE, Rajurkar KP (1992) Stochastic modeling and analysis of abrasive flow machining. ASME J Eng Ind 114:74-81

10. Jain RK, Jain VK, Dixit PM (1999) Modelling of material removal and surface roughness in abrasive flow machining process. Int J Mach Tools Manuf 39:1903-1923, doi:10.1016/ S0890-6955(99)00038-3

11. Singh S, Shan HS, Kumar P (2002) Wear behavior of materials in magnetically assisted abrasive flow machining. J Mater Process Technol 128:155-156, doi:10.1016/S0924-0136(02)00442-9

12. McCarty RW (1970) US Patent 3,521,412

13. Siwert DE (1974) Tooling for the extrude hone process. SME International Engineering Conference, pp 302-315

14. Davies PJ, Fletcher AJ (1995) The assessment of rheological properties of various polyborosiloxane/grit mixtures as utilized in AFM. Proc Inst Mech Engr 209:409-418, doi:10.1243/PIME PROC_1995_209_171_02

15. Rajurkar KP, Kozak J (1999) Hybrid machining process evaluation and development. www.unl.com

16. Jones AR, Hull JB (1998) Ultrasonic flow polishing. Ultrasonics 36:97-101, doi:10.1016/S0041-624X(97)00147-9

17. Gilmore JR, Rhoades LJ (1997) Orbital polishing — an emerging technology. SME, Buff and Polish Clinic, Schaumburg, IL

18. Spur G, Holl SE (1996) Ultrasonic assisted grinding of ceramics. J Mater Process Technol 62(4):287-293, doi:10.1016/S0924-0136 (96)02422-3

19. Takazazawa K, Shinmura T, Hatano E (1985) Development of magnetic abrasive finishing and its equipment. Proceedings of Deburring and Surface Conditioning, SME Conference, Orlando, USA

20. Shinumura T, Yamaguchi H (1995) Study on a new internal finishing process by the application of magnetic abrasive machining. JSME Int J, C 38(4):798-804

21. Kremen GZ, Elsayed EA, Rafalovich VI (1996) Mechanism of material removal in MAP and the accuracy of machining. Int J Prod Res 34(9):2629-2638, doi:10.1080/00207549608905048

22. Khairy AB (2000) Aspects of surface and edge finishing by magneto abrasive particles. Proceedings of International Conference on advanced Manufacturing Technology, Malaysia, pp 77-84

23. Yamaguchi H, Shinmura $T$ (2000) Study of an internal magnetic abrasive finishing using a pole rotation system-discussion of the characteristic abrasive behavior. Precis Eng 24:237-244

24. Singh S, Shan HS (2002) Development of magneto-abrasive flow machining process. Int J Mach Tool Manuf 42m:953-959

25. Fisher RA (1925) Statistical methods for research workers. Oliver and Boyd, London

26. Ross PJ (1988) Taguchi techniques for quality engineering. McGraw-Hill Book Company, New York 
27. Roy RK (1990) A primer on Taguchi method. Van Nostrand Reinhold, New York

28. Walia RS, Shan HS, Kumar P (2004) Analyzing the total number of dynamic active abrasive grains in abrasive flow machining process. NCSAME-2004, JNTU College of Engineering, Hyderabad, 29 to 30 June

29. Walia RS, Shan HS, Kumar P (2006) Parametric optimization of centrifugal force assisted abrasive flow machining
(CFAAFM) By the Taguchi method. Journal of Materials and Manufacturing Processes, USA. Vol. 21, Issues 4 and 5 (available online)

30. Mori T, Hirote K, Kawashime Y (2003) Clarification of magnetic abrasive finishing mechanism. J Mater Process Technol 143144:682-686, doi:10.1016/S0924-0136(03)00410-2

31. Fox RW, Mc Donald AT (1995) Introduction to fluid mechanics. Wiley, New York 